\title{
Ontogenetic changes of trunk muscle structure in the Japanese black salamander (Hynobius nigrescens)
}

\author{
Ayano OMURA ${ }^{1,3) *}$, Wataru ANZAI ${ }^{2,3)}$, Daisuke KOYABU ${ }^{3)}$ and Hideki ENDO ${ }^{3)}$ \\ ${ }^{1)}$ Graduate School of Agricultural and Life Sciences, The University Museum, The University of Tokyo, 7-3-1 Hongo, Bunkyo-ku, Tokyo \\ 113-0033, Japan \\ ${ }^{2)}$ Graduate School of Biological Sciences, The University Museum, The University of Tokyo, 7-3-1 Hongo, Bunkyo-ku, Tokyo 113-0033, \\ Japan \\ ${ }^{3)}$ The University Museum, The University of Tokyo, 7-3-1 Hongo, Bunkyo-ku, Tokyo 113-0033, Japan
}

(Received 7 January 2015/Accepted 13 March 2015/Published online in J-STAGE 30 March 2015)

ABSTRACT. We investigated ontogenetic changes in the trunk muscles of the Japanese black salamander (Hynobius nigrescens) before, during and after metamorphosis. Given that amphibians change their locomotive patterns with metamorphosis, we hypothesized that they may also change the structure of their trunk muscles. The trunk muscles were macroscopically observed, and the weight ratios of each trunk muscle group were quantified at six different developmental stages. Immediately after hatching, we found that the lateral hypaxial muscle was composed of one thick M. ventralis, from ventral edge of which M. transversus abdominis arose later, followed by M. obliquus externus and $M$. rectus abdominis. The weight ratios of the dorsal and abdominal muscles to the trunk muscles increased with growth. We suggest that a single thick and large lateral hypaxial muscle facilitates swimming during early developmental stages. The increase in the weight ratios of the dorsal and abdominal muscles with growth possibly assists with gravity resistance necessary for terrestrial life.

KEY WORDS: Japanese black salamander, locomotion, metamorphosis, trunk muscle

doi: 10.1292/jvms.15-0011; J. Vet. Med. Sci. 77(8): 931-936, 2015

Most species of Urodela ontogenetically change their habitats from water to land [3]. There are 10 families of Urodela which is composed of 670 species. Before metamorphosis, they laterally bend both trunk and tail to swim in water, similar to the locomotion of anguilliforms [8, 23], and well-developed larvae crawl on the bottom of water. In contrast, juveniles of terrestrial species and some semi-aquatic species walk on the ground after completing metamorphosis. In Urodela, to allow for the transition of habitat from water to land, the locomotive structure of the trunk may need to change, because of the need for resistance for viscosity of water in swimming or gravity in terrestrial walking of swimming and walking. Developmental changes in external traits have been studied in various species of Urodela [9]. Of the Japanese Urodela species, Cynops pyrrhogaster [17], Andrias japonicas [11], Hynobius nigrescens [10, 22] and Onychodactylus japonicus [9] have been described. In other species, Ambystoma mexicanum [2] and Pleurodeles walt [20] have been described about developmental changes in external traits. Developmental changes in the inner trunk musculature of Urodela have been examined by Maurer [13] and Fujimoto [7]. Fujimoto [7] described the ontogenetic changes in the trunk muscles of $H$. nebulosus. He showed

*Correspondence to: Omura, A, Graduate School of Agricultural and Life Sciences, The University Museum, The University of Tokyo, 7-3-1 Hongo, Bunkyo-ku, Tokyo 113-0033, Japan. e-mail: ayanodesuyoroshiku@yahoo.co.jp

(C)2015 The Japanese Society of Veterinary Science

This is an open-access article distributed under the terms of the Creative Commons Attribution Non-Commercial No Derivatives (by-nc-nd) License $<$ http://creativecommons.org/licenses/by-nc-nd/3.0/>. that $M$. obliquus internus arises ventrally from the inner ridge of the growth girdle of M. ventralis. M. obliquus externus is then formed dorsally along the end of the ventral surface of $M$. obliquus internus, and $M$. rectus abdominis develops from ventral line. M. subvertebralis and M. transversus abdominis appear after these muscles have developed. Maurer [13] mentioned that all hypaxial muscles constructed body wall were from myomere. He showed that when M. obliquus internus reaches the ventral edges of ventral side of body wall, $M$. rectus abdominis is formed on ventral median line, and M. obliquus externus is formed dorsally. The way of formation of $M$. rectus abdominis and $M$. obliquus externus which arise from the ventral edge is the same between Maurer [13] and Fujimoto [7]. Though Maurer [13] and Fujimoto [7] described the ontogenetic changes in the trunk muscles, they did not discuss the relationships with the shape of the trunk muscles and the habitat. Adult salamanders with different habitats are reported to possess different compositions of trunk muscles according to their habitats [14, 15, 21]. Simons and Brainerd [21] compared four species of adult salamanders and showed that aquatic species possessed a larger total cross-sectional hypaxial area than species that were primarily terrestrial. Omura et al. $[14,15]$ showed that the more aquatic salamanders have larger lateral hypaxial muscles and an unseparated $M$. rectus abdominis for swimming, whereas the more terrestrial species possess larger dorsal muscles and a separated larger $M$. rectus abdominis for resisting gravity. In general, separated muscle has more specialized functions than muscle with unseparated structure from other muscles. Therefore, the more terrestrial species may possess the $M$. rectus abdominis with more specialized function, which is sustaining own weight from downward 
$[14,15]$. Separated muscles are enclosed by fascia of collagen and have intersectio tendinea. It is suggested that the structure may enable the muscle to more strengthen.

Given that muscle patterns vary among salamander species with aquatic or terrestrial habitats, it is possible that muscle patterns may also change according to the different ontogenetic stages because salamanders depend on different habitats throughout growth. Thus, we hypothesized that the ontogenetic changes in the trunk muscles should be correlated with changing locomotion modes from swimming to walking and habitats from water to ground. Parallel to the interspecific patterns found in salamanders [14, 15, 21], we expected that the lateral hypaxial muscles would be larger in the swimming larvae and the dorsal muscles and $M$. rectus abdominis would increase with growth. To test this hypothesis, we observed and quantified the ontogenetic changes in the trunk muscles of $H$. nigrescens. This species changes their habitats and the mode of locomotion by metamorphosis from aquatic swimming larvae to terrestrial walking juvenile. Adult of $H$. nigrescens has relatively larger dorsal muscles, thin lateral hypaxial muscles and separated abdominal muscle to adapt terrestrial life [14].

\section{MATERIALS AND METHODS}

The egg batches of $H$. nigrescens were collected in Niigata Prefecture, Japan, in April 2012. The batches were introduced into an aerated aquarium. After hatching, the larvae were maintained as they metamorphosed through the different developmental stages. Three samples were randomly collected at six developmental stages (Fig. 1 and Table 1), as described by Iwasawa and Yamashita [10]. The earliest stage used in this study was st 38 , which is gill formation III when the gills bud and balancers elongate. The larvae of st 38 swim in water by lateral undulation of trunk. The next developmental stage used in this study was st 50, which is digital differentiation III when the balancers disappear and first and second finger primordials clearly develop. They use their trunk laterally to swim. The third developmental stage used was st 58, which is digital differentiation VI when the fourth toe is clearly recognized. They swim by lateral undulation of trunk and sometimes hold the bottom by forelimbs to stabilize their body. The fourth developmental stage used was st $63 \mathrm{~A}$, which is full-grown larva I when the membrane between each toe disappears. The larvae of st 63A swim using lateral undulation of trunk in addition to crawl on the bottom. The fifth developmental stage was st 66 , which marks the disappearance of fin II when the dorsal fin regresses as far back as the hind limbs and small gill pieces remain. They swim by lateral undulation of trunk and crawl on the bottom. The last developmental stage used was st 68 , which is completion of metamorphosis when the gills and tail fin have completely disappeared and the eyeballs protrude. They land and walk on ground. Samples were fixed in a straight body position in $10 \%$ formalin and were then transferred to $70 \%$ ethanol solution.

The lateral side of the trunk muscles was observed after macroscopic dissection. The following groups of the trunk

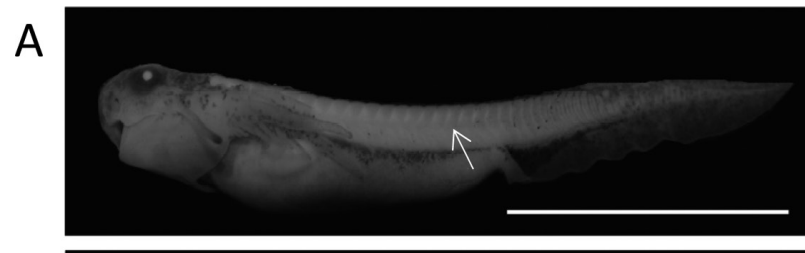

B

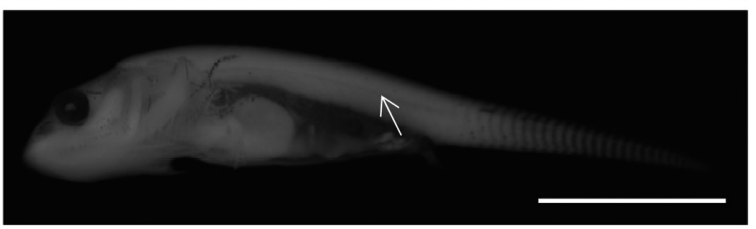

C

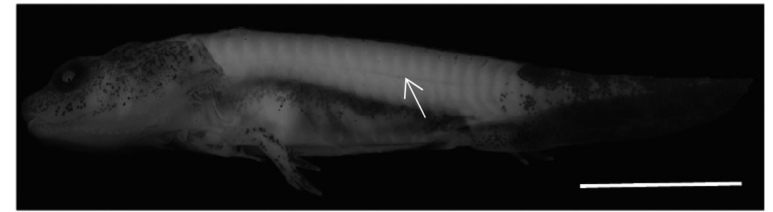

D

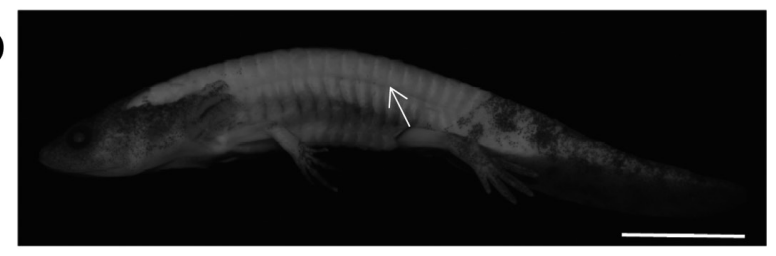

$\mathrm{E}$

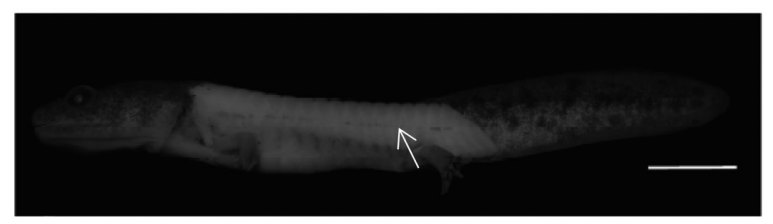

F

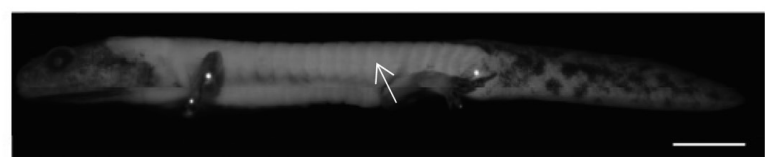

Fig. 1. Lateral views of ontogenetic changes in skinned Hynobius nigrescens. Arrows indicate the level of the line between epaxial muscles and hypaxial muscles. A: st 38, B: st 50, C: st 58, D: st 63A, E: st 66, F: st 68 . Scale bar $=5 \mathrm{~mm}$.

muscles were examined in this study: dorsal muscles, lateral hypaxial muscles and abdominal muscles. During dissection, specimens were kept wet by moistening with water to avoid drying and subsequent measurement error. When the muscle was dried, the weights of the muscle would be measured lighter than in actual, and thus, the assumed measurement error was delivered from the drying of muscles. Each trunk muscle group was weighed using an electronic balance AUW220 (Shimadzu Co., Ltd., Kyoto, Japan). The weight ratio of each muscle group against the total weight of all measured trunk muscles was calculated.

\section{RESULTS}

Observation of trunk muscles: The components of the trunk muscles developed and changed morphologically with growth (Fig. 2). At st 38, they possessed a single thick dorsal 
Table 1. Specimens of Hynobius nigrescens used in this study

\begin{tabular}{ccc}
\hline Developmental stage & Habitat & SVL*(mm) \\
\hline st38 & Aquatic & 7.2 \\
& & 7.3 \\
\multirow{2}{*}{ st50 } & Aquatic & 9.5 \\
& & 10.9 \\
& & 10.8 \\
st58 & Aquatic & 12.9 \\
& & 13.5 \\
& & 14.1 \\
st63A & Aquatic & 16.8 \\
& & 17.1 \\
& & 17.2 \\
st66 & Aquatic & 21.1 \\
& & 21.6 \\
& & 22.2 \\
st68 & Terrestrial & 24.9 \\
& & 25.2 \\
& & 25.7 \\
\hline
\end{tabular}

*Snout-vent length.

muscle and a single thick M. ventralis (Fig. 2A). At st 50, when the first and second finger primordia had developed patently, a thin $M$. transversus abdominis with fibers extending craniodorsally developed from $M$. ventralis and became ventrally enlarged (Fig. 2B). M. ventralis became thinner at st 50 . The dorsal muscles were segmented by myosepta, as also observed at st 38 . When the hind limbs were revealed and forelimbs were developed at st 58, a thin M. obliquus externus with fibers running caudoventrally developed along the edge of the abdominal contour line of $M$. transversus abdominis. From the ventral edge of M. obliquus externus, the muscle fibers of $M$. obliquus externus became parallel to the sagittal line (Fig. 2C). At st 63A, when they crawled on the bottom in water and swam by undulation, M. obliquus externus dorsally developed to the level of the lateral line between epaxial muscles and hypaxial muscles (Fig. 2D). A thin $M$. rectus abdominis occurred at the ventral edge of the trunk (Fig. 2D). At st 66, when they crawled on the bottom in water in addition to swimming, $M$. rectus abdominis expanded and increased in thickness and was obviously separated from the fibers of the lateral hypaxial layers (Fig. $2 \mathrm{E})$. At st 68 , after metamorphosis and movement to land for walking on ground, $M$. rectus abdominis became enlarged (Fig. 2F) and became thicker.

Trunk muscle weight ratios: The muscle group weight ratios are represented in Table 2. Ontogenetic changes were identified in the muscle group ratios among stages. The muscle weight ratio of the dorsal muscles increased with growth from $52.2 \%$ at st 38 to $61.4 \%$ at st 68 (the averages of three samples of weight ratios of the trunk muscles: Table 2). In contrast, the weight ratio of the lateral hypaxial muscles decreased with growth from $>40 \%$ at st 38,50 and 58 to $<30 \%$ at st 66 (Table 2). The weight ratio of the abdominal muscles increased with growth (Table 2). At st 38 and 50, the salamander did not possess abdominal muscles, with the percentage of abdominal muscles being recorded as zero. From st 58 to 68 , the muscle weight ratio of the abdominal muscles increased from 3.0 to $16.5 \%$.

\section{DISCUSSION}

Observation of trunk muscles: Ontogenetic changes were recognized in the trunk muscles in $H$. nigrescens. Maurer [13] described that $M$. rectus abdominis arose from the ventral edges of $M$. obliquus internus on the timing of start of development of $M$. obliquus externus. In H. nebulosus, $M$. rectus abdominis develops from the ventral edges of $M$. obliquus externus and M. obliquus internus when the development of M. obliquus externus starts [7]. In this study of $H$. nigrescens, $M$. rectus abdominis developed and enlarged from the ventral line when $M$. obliquus externus developed. The timing of formation of $M$. rectus abdominis on the ventral line in this study coincided with Maurer [13] and Fujimoto [7].

The number of lateral hypaxial muscles differed between $H$. nigrescens in this study and $H$. nebulosus in Fujimoto [7]. H. nigrescens has two layers, M. obliquus externus and $M$. transversus abdominis, as lateral hypaxial muscles, except for $M$. ventralis [7]. In contrast, $H$. nebulosus has three layers: M. obliquus externus, M. obliquus internus and $M$. transversus abdominis, except for $M$. ventralis. The developmental sequence of the hypaxial trunk muscles of $H$. nebulosus is reported as follows: 1) M. ventralis (the ventral muscle), 2) M. obliquus internus (the inner lateral hypaxial layer) from the ventral muscle, 3) M. obliquus externus (the outer lateral hypaxial layer) and $M$. rectus abdominis and 4) $M$. transversus abdominis [7], which sequence was similarly observed in $H$. nigrescens, but $M$. obliquus internus does not appear and $M$. transversus abominis appears before $M$. obliquus externus and $M$. rectus abdominis in $H$. nigrescens. Simons and Brainerd [21] discussed that the habitat and predominant locomotor mode of salamanders do not appear to be strong associated with the number of lateral hypaxial layers. It was suggested that the differences in the number of lateral hypaxial layers in these phylogenetically very close species, $H$. nebulosus and $H$. nigrescens suggest the presence of interspecific variation in this genus and further studies on other congeneric species are required. In this study, we observed only from lateral view. Then, $M$. subvertebralis was not examined. In adult of $H$. nigrescens, $M$. subvertebralis exists under vertebrae [14].

At st 38, immediate hatching, the number of lateral hypaxial layer was only one, and the layer was thick (Fig. 2A). A typical fish possesses a thick trunk muscle divided into epaxial and hypaxial segments by a myosepta, but it does not show a layered structure [12]. Because the larvae of $H$. nigrescens locomote by swimming and they do not possess limbs at st 38 , they have a single thick $M$. ventralis as the lateral hypaxial muscle for undulatory swimming. During later developmental stages, the thickness of $M$. ventralis decreased, and a thin layer of $M$. transversus abdominis developed from the ventral edge of M. ventralis (Fig. 2B). 
A

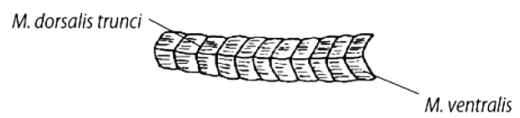

B

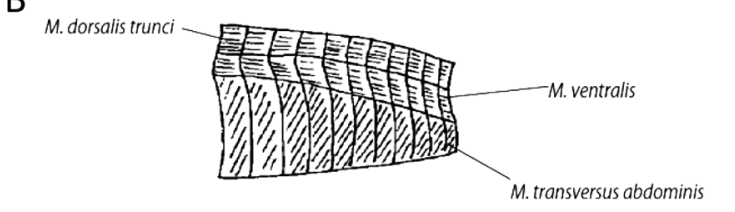

C
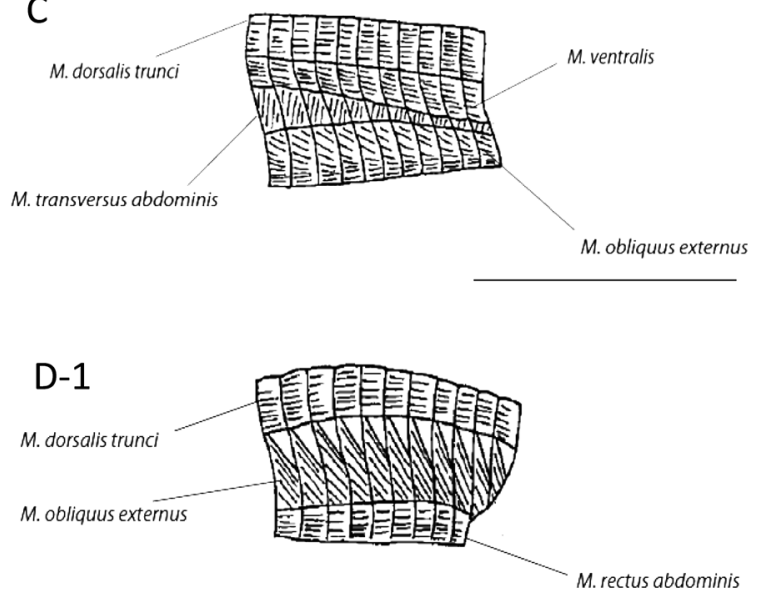

\section{D-2}

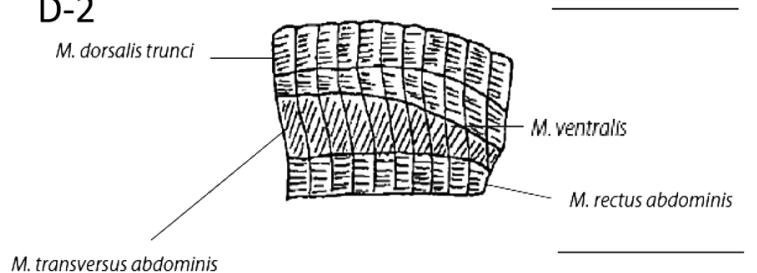

E-1

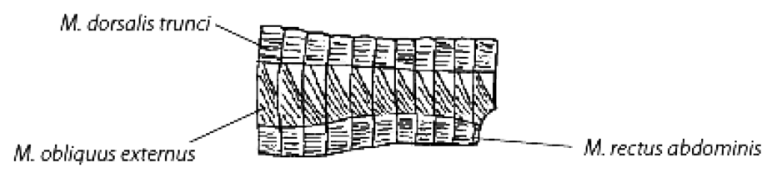

E-2

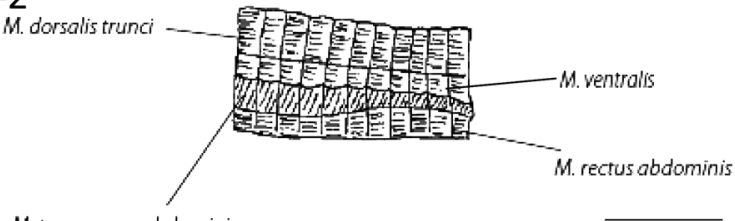

M. transwersus abdominis

\section{F-1}

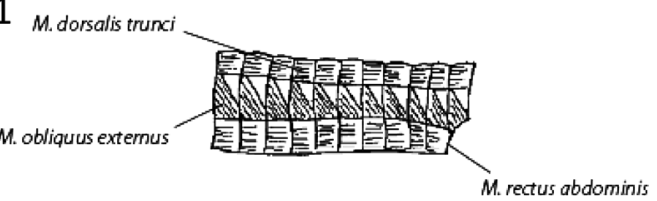

F-2

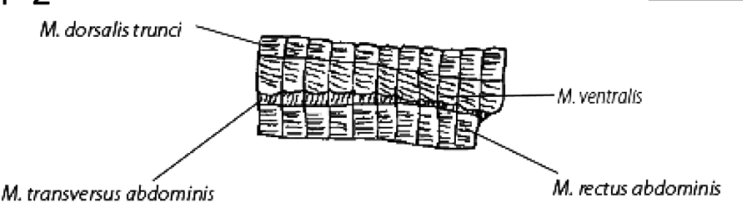

Fig. 2. Lateral views of ontogenetic changes of the trunk muscles in Hynobius nigrescens. A: st 38, B: st 50, C: st 58, D-1: st 63A after skinned, D-2: st 63A after removed M. obliquus externus, E-1: st 66 after skinned, E-2; st 66 after removed M. obliquus externus, F-1: st 68 after skinned, F-2: st 68 after removed M. obliquus externus. Scale bar $=5 \mathrm{~mm}$.

Table 2. Ontogenetic changes of weight ratios of trunk muscles in H. nigrescens

\begin{tabular}{lcccc}
\hline \multirow{2}{*}{$\begin{array}{c}\text { Developmental } \\
\text { stage }\end{array}$} & $\begin{array}{c}\text { Averages of SVL* } \\
(\mathrm{mm})\end{array}$ & \multicolumn{3}{c}{ Weight ratio (\%) } \\
\cline { 3 - 5 } & $7.33 \pm 0.2$ & $52.2 \pm 2.3$ & $47.7 \pm 2.3$ & Dorsal muscles \\
\hline st38 & $10.4 \pm 0.3$ & $55.1 \pm 2.3$ & $44.8 \pm 2.3$ & 0.0 \\
st50 & $13.5 \pm 1.1$ & $55.3 \pm 1.8$ & $41.7 \pm 2.2$ & $3.0 \pm 0.4$ \\
st58 & $17.0 \pm 0.6$ & $56.7 \pm 2.6$ & $38.3 \pm 2.9$ & $4.9 \pm 0.3$ \\
st63A & $21.6 \pm 1.3$ & $60.0 \pm 1.2$ & $29.9 \pm 2.4$ & $10.0 \pm 1.3$ \\
st66 & $25.2 \pm 1.2$ & $61.4 \pm 2.3$ & $22.1 \pm 3.5$ & $16.5 \pm 1.4$ \\
st68 & & & & \\
\hline
\end{tabular}

*Snout-vent length, mean \pm S.E.M.

At st 58, a thin layer of $M$. obliquus externus developed as one of the lateral hypaxial muscles (Fig. 2C). The two lateral hypaxial muscles at this stage were thinner than $M$. ventralis present during early developmental stages (Fig. 2C). When Urodelians move to land, they need to resist both torsion and lateral bending [3]. Since resisting torsion can be absorbed 
by the two lateral hypaxial layers which fibers run in a cross direction with each other, the number of the lateral hypaxial muscles possibly increased from one to two with growth. Furthermore, muscle fibers running across each other in the two lateral hypaxial layers strengthen the body in a manner similar to the lamination of chipboard [12]. At st 58, when the fourth toe is clearly recognized, the muscle fibers of the lateral hypaxial muscle become longitudinal at the ventral edge of the trunk (Fig. 2C). M. rectus abdominis developed and enlarged at st 58, 63A, 66 and 68 (Fig. 2C-2F).

Because $M$. rectus abdominis generally contributes to maintaining posture [4] and sustaining the animals' own weight [18], evolutionary acquisition of this muscle was possibly essential for terrestrial locomotion. It has been argued that typical fish do not have $M$. rectus abdominis [12] because their basic trunk muscle structure is composed of epaxial and hypaxial muscles that facilitate lateral bending. Because of buoyancy, the need of sustaining inward organs weight decreases in fish. Then, fish does not have $M$. rectus abdominis which function is sustaining own weight. When adult salamanders are compared, terrestrial species and semi-aquatic species possess a separated and larger $M$. rectus abdominis, whereas aquatic species possess a smaller and unseparated $M$. rectus abdominis $[14,15]$. Omura et al. $[14,15]$ suggested that a separated and larger $M$. rectus $a b-$ dominis facilitated terrestrial locomotion by resisting gravity and that $M$. rectus abdominis is not essential for an aquatic lifestyle. Separated $M$. rectus abdominis more specializes its function of sustaining own weight than unseparated ones from lateral hypaxial muscles $[14,15]$. Separated $M$. rectus abdominis possesses collagen fascia and intersection tendinea. The fascia and intersection tendinea may enable the muscle to more strengthen. Thus, the interspecific difference of $M$. rectus abdominis between aquatic and terrestrial species is parallel to the muscular differences found between the aquatic and terrestrial stages of $H$. nigrescens.

Trunk muscle weight ratios: Ontogenetic changes in the weight and weight ratios of the muscle groups are given in Table 2. Though the actual mass of all muscle groups increased according to growth, the degree of growth was different among muscle groups. The weight ratios of the dorsal and abdominal muscles increased with growth, hence the decrease of the weight ratios of the lateral hypaxial muscles. Epaxial muscles are recruited for lateral bending during swimming and walking [4-6]. Dorsal muscles also function as stabilizers of the trunk [16]. M. dorsalis trunci, which is the largest epaxial muscle, stabilizes the trunk against sagging and torsion and increases the stiffness of the trunk during walking [4]. Because the need for stabilizing the trunk and resisting gravity on land occurs only after metamorphosis, we assume that the increase in the weight ratio of the dorsal muscles is related to the transition from water to land.

The weight ratio of the lateral hypaxial muscles decreased with growth (Table 2). Lateral hypaxial muscles function to control torsion and lateral bending [19] and to stabilize the body $[1,3,4]$. Because lateral hypaxial muscles are necessary for undulatory swimming, we suggest that larvae possess larger lateral hypaxial muscles than terrestrial juve- niles. After the limbs develop, the role of the lateral hypaxial muscles possibly decreases. Therefore, we consider that the decreased importance of the lateral hypaxial muscles results in the decreased weight ratios of the lateral hypaxial muscles. After they are equipped with limbs, Urodelians mainly locomote by undulatory swimming in addition to aquatic walking. This study suggests that they gradually modify the trunk muscles to prepare for movement on land.

The weight ratio of the abdominal muscles increased with growth (Table 2). Abdominal muscles function to prevent sagittal extension of the trunk by the action of the epaxial muscles [4] and to sustain the body weight against gravity [18]. Abdominal muscles appeared and increased in size after the appearance of the interdigital processes in the hind limb anlage. It is possible that such growth of the abdominal muscles facilitates terrestrial life. During middle developmental stages from st 50 to st $63 \mathrm{~A}$, salamanders start swimming in water using their limbs (personal observations). At this stage, they depend less on the lateral hypaxial muscles and more on the dorsal and abdominal muscles. After metamorphosis, they start adapting to terrestrial life by enlarging the dorsal and abdominal muscles.

In conclusion, the ontogenetic changes in the trunk muscles of $H$. nigrescens are linked with habitat transition from water to land, with the muscle construction changing in adaptation from aquatic swimming to resisting gravity.

ACKNOWLEDGMENTS. We appreciate Dr. Shin-ichiro Kawada of National Museum of Science and Nature, Tokyo, for giving us some specimens. We also thank Dr. Natsuhiko Yoshikawa of Kyoto University, Dr. Yukio Aizawa of Nippon Dental University and Dr. Kenjiro Kinebuchi of Niigata Seiryo University for providing Hynobius nigrescens specimens. We thank Dr. Takenori Sasaki of The University Museum, The University of Tokyo and Mr. Takashi Yoshimine of Microscope Network Co., Ltd. for providing some equipment for functional morphological analysis. The research was financially supported by the Sasakawa Scientific Research Grant (No. 25-539) from The Japan Science Society.

\section{REFERENCES}

1. Bennett, W. O., Simons, R. S. and Brainerd, E. L. 2001. Twisting and bending: the functional role of salamander lateral hypaxial musculature during locomotion. J. Exp. Biol. 204: 1979-1989. [Medline]

2. Bordzilovskaya, N. P. and Dettlaff, T. A. 1979. Table of stages of the normal development of axolotl embryos. Smith. VII.

3. Carrier, D. R. 1993. Action of the hypaxial muscles during walking and swimming in the salamander Dicamptodon ensatus. $J$. Exp. Biol. 180: 75-83.

4. Deban, S. M. and Schilling, N. 2009. Activity of trunk muscles during aquatic and terrestrial locomotion in Ambystoma maculatum. J. Exp. Biol. 212: 2949-2959. [Medline] [CrossRef]

5. Delvolvé, I., Bem, T. and Cabelguen, J. M. 1997. Epaxial and limb muscle activity during swimming and terrestrial stepping in the adult newt, Pleurodeles waltl. J. Neurophysiol. 78: 638-650. [Medline]

6. Frolich, L. M. and Biewener, A. A. 1992. Kinematic and elec- 
tromyographic analysis of the functional role of the body axis during terrestrial and aquatic locomotion in the salamander Ambystoma tigrinum. J. Exp. Biol. 162: 107-130.

7. Fujimoto, T. 1960. Development of the adbominal musculature of Hynobius nebulosus. J. Okayama Med. Assoc. 611: 22052220 (in Japanese).

8. Hoff, K. S., Hug, N., King, V. A. and Wassersug, R. J. 1989. The kinematics of larval salamander swimming (Ambystomatidae: Caudata). Can. J. Zool. 67: 2756-2761. [CrossRef]

9. Iwasawa, H. and Kera, Y. 1980. Normal stages of development of the Japanese lungless salamander, Onychodactylus japonicus (Houttuyn). Jpn. J. Herpetol. 8: 73-89 (in Japanese).

10. Iwasawa, H. and Yamashita, K. 1991. Normal stages of development of hynobiid salamander, Hynobius nigrescens Stejneger. Jpn. J. Herpetol. 14: 39-62 (in Japanese).

11. Kudo, T. 1938. Normentafel zur Entwicklungsgeschichte des japanischen Riesensalamanders (Megalobatrachus japonicus Temminck). Normen. Ent. Wirbel. 16: 98.

12. Liem, K. F., Walker, W. F., Bemis, W. E. and Grande, L. 2001. Functional Anatomy of the Vertebrates: An Evolutionary Perspective. Harcourt College Press, Philadelphia.

13. Maurer, F. 1892. Der Aufbau und die Entwicklung der ventralen Rumphmuskulatur bei den urodelen Amphibien und deren Beziehungen zu den gleichen Muskeln der Selachier und Teleostier. Morphol. Jahrb. 18: 76-179.

14. Omura, A., Anzai, W. and Endo, H. 2014. Functional and morphological variety in trunk muscles of Urodela. J. Vet. Med. Sci.
76: 159-167. [Medline] [CrossRef]

15. Omura, A., Ejima, K., Honda, K., Anzai, W., Taguchi, Y., Koyabu, D. and Endo, H. 2015. Locomotion pattern and trunk musculoskeletal architecture among Urodela. Acta Zool. online open, in press. [CrossRef]

16. O'Reilly, J. C., Summers, A. P. and Ritter, D. A. 2000. The evolution of the functional role of trunk muscles during locomotion in adult amphibians. Am. Zool. 40: 123-135. [CrossRef]

17. Oyama, J. 1930. Normal stages of development of Japanese newt. Zoolog. Sci. 42: 465-473 (in Japanese).

18. Preuschoft, H., Schulte, D., Distler, C., Witzel, U. and Hohn, B. 2007. Body shape and locomotion in monitor lizards. Mertensiella 16: 59-78.

19. Schilling, N. 2011. Evolution of the axial system in craniates: morphology and function of the perivertebral musculature. Front. Zool. 8: 4-22. [Medline] [CrossRef]

20. Shi, D. L. and Boucaut, J. C. 1995. The chronological development of the urodele amphibian Pleurodeles waltl (Michah). Int. J. Dev. Biol. 39: 427-441. [Medline]

21. Simons, R. S. and Brainerd, E. L. 1999. Morphological variation of hypaxial musculature in salamanders (Lissamphibia: caudata). J. Morphol. 241: 153-164. [Medline] [CrossRef]

22. Usui, M. and Hamasaki, M. 1939. Normal stages of development of Hynobius nigrescens. Zoolog. Sci. 51: 195-206 (in Japanese).

23. Wassersug, R. J. 1989. Locomotion in amphibian larvae (or “why aren't tadopoles built like fishes?”). Am. Zool. 29: 65-84. 